\title{
The Small-Angle Scattering and HPLC-SAXS modules of the US-SOMO software suite
}

\author{
$\underline{\text { Mattia Rocco, }},{ }^{\mathrm{a}}$ Javier Pérez, ${ }^{\mathrm{b}}$ Patrice Vachette, ${ }^{\mathrm{c}}$ Emre Brookes ${ }^{\mathrm{d}}$ \\ ${ }^{a}$ Biopolimeri e Proteomica, Ospedale Policlinico San Martino, Genova, Italy.mattia.rocco@ hsanmartino.it; \\ ${ }^{b}$ Beamline SWING, Synchrotron SOLEIL, Gif-sur-Yvette, France; ${ }^{c} I 2 B C$, CEA, CNRS, Université Paris-Sud, \\ Université Paris-Saclay, Gif-sur-Yvette, France; Dept. of Biochemistry, University of Texas at San Antonio Health \\ Center, San Antonio, TX, USA ${ }^{d}$
}

The UltraScan SOlution MOdeller (US-SOMO) is a comprehensive, public domain, open-source suite of computer programs centred on hydrodynamic modelling and small-angle scattering (SAS) data analysis and simulation [1]. US-SOMO is a GUI application freely available either as source code or as precompiled executables for Windows, MAC/OSX and Linux (http://www.sas.uthscsa.edu/). USSOMO provides a unified environment and includes a variety of SAS analysis tools. SAS functions include the ability to compute $I(q)$ vs. $q$ curves from atomic coordinates utilizing a variety of methods, including CRYSOL, CRYSON, FOXS, as well as our own full Debye, spherical harmonic and fast methods. Fitting of multiple model curves to experimental data is available with best fit and nonnegative least squares methods. Batch modes provide the ability to compute $I(q)$ profiles on multiple structures. Guinier analysis methods include transverse and cross-sectional variants. Molecular mass, mass/length and mass/area can be correctly computed from known standards, and the mass can be approximated utilizing the Rambo-Tainer [2] method. Along with multiple $P(r)$ vs. $r$ computational tools, the Indirect Fourier Transform (IFT) using Bayesian estimation via the BayesApp [3] method has been incorporated. SAXS-coupled size exclusion chromatography (SEC) experiments are supported by the HPLC module [4,5]. Included are a physics based method for capillary fouling correction and a $P$-value pair map tool to assess the identity of a set of curves based on the Cormap approach [6]. A time-collection of $I_{t}(q)$ vs. $q$ frames can be easily converted into a collection of $I_{q}(t)$ vs. $t$ curves (Fig. 1A-B). Transforming not baseline resolved SEC-SAXS data into separate monodisperse equivalents is supported by Gaussian decompositions (Fig. 1C), from which separate $I_{t}(q)$ vs. $q$ frames can be reconstructed for each peak. Concentration profiles can be simultaneously decomposed to assist in computing accurate molecular masses. The example provided in Fig. 1A-C reports the SEC-SAXS analysis of supramolecular complexes formed by the aldolase tetramer in solution. The SAXS curves of the decomposed peaks and their IFT-derived $P(r)$ vs. $r$ are then used for molecular modeling.
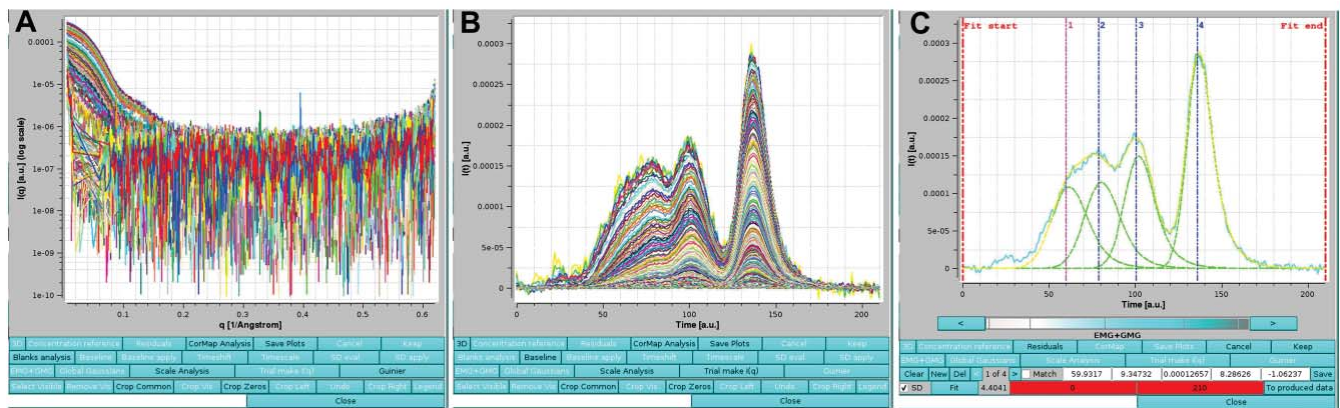

Figure 1. Conversion of a series of $I_{t}(q)$ SEC-SAXS frames (A) into a series of $I_{q}(t)$ chromatograms (B), and their Gaussian decomposition into 4 separate peaks (C, only one chromatogram is shown).

[1] E. H. Brookes, M. Rocco Eur. Biophys. J. 2018, in press (DOI: 10.1007/s00249-018-1296-0).

[2] R. P. Rambo, J. A. Tainer Nature 2013, 496, 477.

[3] S. Hansen J. Appl. Cryst. 2000, 33, 1415.

[4] E. H. Brookes et al J. Appl. Cryst. 2013, 46, 1823.

[5] E. H. Brookes et al J. Appl. Cryst. 2016, 49, 1827.

[6] D. Franke et al. Nature Methods 2015, 12, 419. 